\title{
PRELIMINARY RESULTS OF DETERMINISTIC CHAOS CONTROL THROUGH COMPLEXITY MEASURES
}

\author{
Eduard Navratil \\ Ivan Zelinka \\ Roman Senkerik \\ Department of Applied Informatics \\ Faculty of Applied Informatics \\ Tomas Bata University in Zlin \\ Nad Stranemi 4511, Zlin 76005, Czech Republic \\ enavratil@fai.utb.cz,zelinka@fai.utb.cz,senkerik@fai.utb.cz
}

\begin{abstract}
KEYWORDS
spatiotemporal chaos, coupled map lattices, evolution, optimisation, SOMA, complexity measures, mean mutual information, mean information gain
\end{abstract}

\section{ABSTRACT}

This contribution deals with the application of complexity measures as alternative cost functions for the control of a spatiotemporal deterministic chaos represented by coupled map lattices. Mean mutual information and mean information gain is applied, along with a common 1-norm based cost function. Optimization is done through the self-organizing migrating algorithm. Aim of this study is to see whether the specific properties of the above measures can be exploited in spatiotemporal deterministic chaos control.
\end{abstract}

\section{INTRODUCTION}

The term deterministic chaos control (DCC) was first coined in (Ott E., Greboki C., Yorke J.A., 1990). It represents a process in which such a control law is derived and used that the originally chaotic process would stabilize itself on a constant level of output value or in an n-periodic cycle. Since the first experiments of DCC many methods how to derive control law were developed and based on the first one (Ott E., Greboki C., Yorke J.A., 1990) like pole placement (Greboki C., Lai Y.C. 1999), delay feedback etc. Many of published methods were adapted for so-called spatiotemporal chaos represented by coupled map lattices (CML), given by equation (1). Models of this kind are based on a set of spatiotemporal (for 1D, Figure 1) or spatial cells, which represent the appropriate state of system elements. The typical example is a CML based on the logistic equation (Hilborn R.C., 1994; Guanrong Chen, 2000) which is used to simulate the behaviour of a system consisting of $n$ mutually joined cells - logistic equations.

$$
x_{n+1}(i)=(1-\epsilon) f\left(x_{n}(i)\right)+\frac{\epsilon}{2}\left(f\left(x_{n}(i-1)\right)+f\left(x_{n}(i+1)\right)\right)
$$

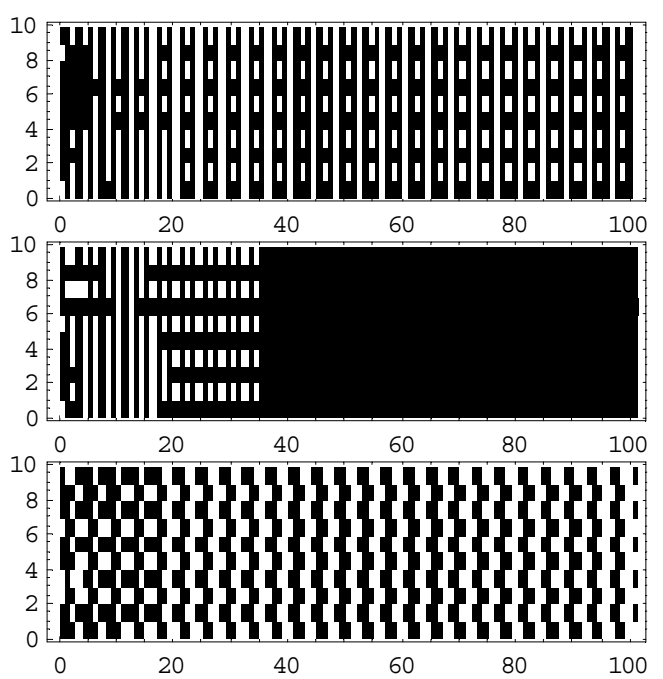

Figure 1: Various kinds of 1D CML behaviour

Although the formulae for both mean mutual information and mean information gain can be found already in the fundamental work of information theory (Shannon C. E., 1948) it was only later that they were recognized and used as a measures of statistical and structural complexity (Wackerbauer et al., 1994). There are many examples of their use: describing the complexity of a logistic equation (Wackerbauer et al., 1994), estimating the complexity of 2D patterns through mean information gain (Andrienko et al., 2000), estimating long range correlations (Li W., 1990) etc.

\section{METHODS}

Means of experiment and their particular settings are described in this section.

\subsection{CML}

The class of CML problems chosen for this study was based on case studies reported in (Schuster H.G., 1999). In the frame of this contribution, CML control means setting of such pinning values (PV, control values) that the system stabilizes itself on an expected spatiotemporal pattern. CML as an object of study was chosen because it shows chaotic behaviour and its level of complexity can be quite high.

The controlled CML consisted of 10 mutually joined logistic equations (1) with periodic boundary. Pinning sites were at each odd lattice, i.e. at each odd lattice the 
control parameter $A$ was set to a pinning value. At even lattices $A$ equalled 4 and $\varepsilon$ was set to 0.8 , which led to a fixed point of a map at $x=0.75$.

\subsection{SOMA}

SOMA (self-organizing migrating algorithm) is a stochastic optimization algorithm that imitates the social behaviour of cooperating individuals. It was chosen due to its ability to converge towards the global optimum (Zelinka Ivan, 2004). SOMA works on a population of candidate solutions in loops called migration loops. In the beginning, the population is randomly distributed over the search space. In each loop, the population is evaluated and the solution with the highest fitness becomes the leader. Apart from the leader, in one migration loop all individuals will traverse the input space in the direction of the leader. Mutation, the random perturbation of individuals, is an important operation for evolutionary strategies. It ensures the diversity of individuals and it also provides means to restore lost information in a population. Mutation is different in SOMA compared with other strategies: SOMA uses a parameter called PRT to achieve perturbation. This parameter has the same effect for SOMA as mutation has for genetic algorithms - the randomly generated binary perturbation vector controls the allowed dimensions of an individual. If an element of the perturbation vector is set to zero, then the individual is not allowed to change its position in the corresponding dimension.

An individual will travel a certain distance (called the path length) towards the leader in $n$ steps of a defined length. If the path length is chosen to be greater than one, then the individual will overshoot the leader. This path is perturbed randomly.

For an exact description of the algorithm see (Zelinka Ivan, 2004). Table 1 gives the control parameter settings.

Table 1: SOMA setting for parts A, B, C

\begin{tabular}{|l|l|l|l|}
\hline & \multicolumn{1}{|c|}{ A } & \multicolumn{1}{c|}{ B } & \multicolumn{1}{c|}{ C } \\
\hline PathLength & 3 & 3 & 3 \\
\hline Step & 0.11 & 0.11 & 0.11 \\
\hline PRT & 0.1 & 0.1 & 0.1 \\
\hline PopSize & 25 & 25 & 25 \\
\hline Migrations & 50 & 20 & 10 \\
\hline MinDiv & -1.0 & -1.0 & -1.0 \\
\hline CF Evaluations & 1900 & 1900 & 1900 \\
\hline
\end{tabular}

\subsection{Discretization of the State Space}

To use the complexity measures the state space A of a single logistic equation has been divided into $n$ cells $A_{1}, . ., A_{n}$ of equal volume with respect to the Lebesguemeasure; both measures then work with a sequence of integers from the set $\{0, . ., n-1\}$. This kind of partition is easy to handle and independent of any specific properties of the system.

In the following experiments the state space of each logistic equation was divided into 100 cells. The number of possible states can be even higher to achieve better precision, however, at the expense of growing memory and calculation time requirements.

\subsection{Mean Information Gain $<$ G $>$}

The information gain $G_{i j}$ represents the information needed for a correct selection of state $A_{j}$ if state $A_{i}$ is known. Mean information gain $<\mathrm{G}>$ is then the average of $G_{i j}$ over all possible transitions from state $A_{i}$ to $A_{j}$, usually described by the following equation (2),

$$
<G>=-\sum_{i, j} p(i, j) \log _{2} p(i \rightarrow j)
$$

where $p(i, j)$ denotes the joint probability of states $\mathrm{A}_{\mathrm{i}}, \mathrm{A}_{\mathrm{j}}$ respectively, and $p(i \rightarrow j)$ stands for the conditional probability. Mean information gain is sensitive to any structural irregularities and reaches 0 only when the sequence is either constant or strictly periodic. On the other hand, $<\mathrm{G}>$ does not discriminate the order of the period; it is only capable of distinguishing a periodical structure from aperiodic one (Wackerbauer et al., 1994).

\subsection{Mean Mutual Information $<M>$}

The mutual information $\mathrm{M}_{\mathrm{ij}}$ can be interpreted as a measure of dependence between two given states $i$ and $\mathrm{j}$; it is zero if the two states are independent. Mean mutual information $<\mathrm{M}>$ (3) is then the average of mutual information $\mathrm{M}_{\mathrm{ij}}$ over all states $\mathrm{i}, \mathrm{j}$.

$$
\begin{aligned}
& <M>=-\sum_{i, j} p(i, j) \log _{2} \frac{p(i, j)}{p(i) p(j)} \\
& <M(x)>=I(x)-<G(x)>
\end{aligned}
$$

where $p(i), p(j)$ represent the probabilities of states $i, j$ respectively, $I(x)$ is the entropy of a given source calculated from the chain of states. Mean mutual information $<\mathrm{M}>$ has one great advantage when compared to $<\mathrm{G}>$, which can be seen from Eq. (4): in case of periodical structure $(<\mathrm{G}>=0)$ it returns only $I(x)$, i.e. the entropy of a source with equally frequent values - the binary logarithm of the period order (Wackerbauer et al., 1994).

\subsection{Cost Functions}

Four cost functions were used in the experiment. Three were using a target pattern: functions based on distance, mean information gain and mean mutual information; the fourth fitness based on $\langle\mathrm{M}>$ was not using any target pattern. All four functions work with the CML output with random initial values. The distance based fitness has been calculated as the 1-norm distance between desired CML state and actual CML output (5). The minimum of this cost function, guaranteeing the best solution, is 0 . Indexes $i$ and $j$ are coordinates of lattice element, i.e. $\mathrm{CML}_{\mathrm{i}, \mathrm{j}}$ is $i^{\text {th }}$ site (equation) in $j^{\text {th }}$ iteration. The target of control, $\mathrm{TS}_{\mathrm{i}, \mathrm{j}}$, was set to 0.75 in all simulations, i.e. CML behaviour was controlled to this simplest state. 


$$
\begin{aligned}
& f_{\text {cos } t}=\sum_{i=1}^{10} \sum_{j=9}^{10}\left|T S_{i, j}-C M L_{i, j}\right| \\
& \mathrm{TS}_{\mathrm{i}, \mathrm{j}} \text { - target state of CML } \\
& \mathrm{CML}_{\mathrm{i}, \mathrm{j}} \text { - actual state of controlled CML }
\end{aligned}
$$

The same function was also used for the evaluation of the fitness of optimization results, save for the different values of $j, 99$ to 100 .

The cost function using mean information gain is quite different (6); instead of measuring the distance from the target pattern it calculates $<\mathrm{G}>$ of the discretized tenth iteration with appended vector of target values (i.e. ten values of 75 in this case). As $<\mathrm{G}>$ yields 0 only for constant or a strictly periodic sequences it can return 0 only when all twenty numbers in the sequence are equal to 75 .

$$
f_{\cos t}=<G\left(C M L_{10}, T S_{10}\right)>
$$

The mean mutual information-based cost function (7) works with the same ordered set of CML and target pattern values and reaches its minimum, i.e. 0 , on the same conditions as $<\mathrm{G}>$-based cost function. When optimizing with no target pattern given, another iteration of CML is used instead of the target line (8).

$$
\begin{aligned}
& f_{\cos t}=<M\left(C M L_{10}, T S_{10}\right)> \\
& f_{\cos t}=<M\left(C M L_{10}, C M L_{11}\right)>
\end{aligned}
$$

\subsection{Experiment Design}

The experiment was divided into three similar parts differing by the number of migration loops. In each of them the cost functions based on the complexity measures and a distance-based fitness were applied in order to bring the CML to an a priori known stable state through pinning value optimization by SOMA; in addition, mean mutual information was used to stabilize the lattices in both time and space without any prior knowledge of the stable state. Each SOMA optimization returned 25 pinning values which were then applied on 100 random-generated initial values and were assigned the mean distance of controlled CML from the desired state. These populations -4 for each of the three parts were then characterized by the least, the average and the greatest distance from the stable state.

\section{EXPERIMENTAL RESULTS}

Above mentioned cost functions were used by SOMA with 10, 20 and 50 migrations. Each individual of the final population was assigned the mean distance of the last two from a hundred iterations; this distance was measured for 100 random initial setups of CML. This yielded the estimation of the best, the worst and the mean error of each SOMA population. In CML control demonstrations white colour appears on lattices where $x_{j}(i)>0.75$.

\subsection{Cost Functions Dependence on Pinning Values}

To see the average dependence on pinning values, a cost function was evaluated ten times for every pinning value. As the number of measurements is quite small the graphs have rather an exemplificative meaning. It should be also kept in mind that all cost functions are evaluated after 10 iterations of CML and may have a different dependence with growing number of iterations.

The cost values of 1-norm based cost function (Figure 2) are not surprising if compared to (Zelinka Ivan, 2005) - the global minimum is situated between pinning values of 2.5 and 3 , fitness close to zero is reached even after ten iterations.

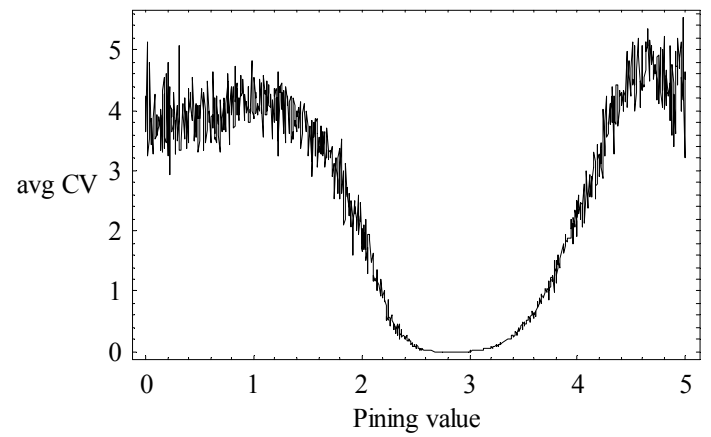

Figure 2: Fitness Dependence on Pinning Values for 1norm Based Cost Function

The mean information gain based cost function has considerably more complicated development, see (Figure 3). Although the region between values of 2 and 4 contains some local minima, the global minimum of this curve can be seen around 0.5 . This graph slightly anticipates the quality of $<\mathrm{G}>$-controlled $\mathrm{CML}$.

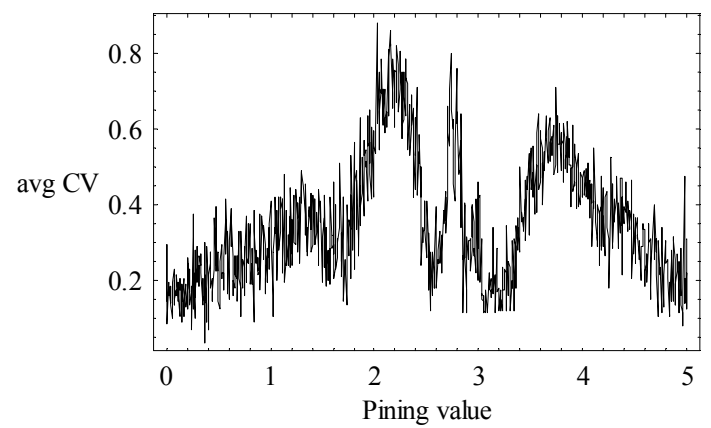

Figure 3: Fitness Dependence on Pinning Values for $<\mathrm{G}>$-based Cost Function

The development of $<\mathrm{M}>$-based fitness (Figure 4) is more like the first graph, with the global minimum in the expected region of pinning values. The cost function with mean mutual information, which was not using a target value, has a similar shape (Figure 5). 


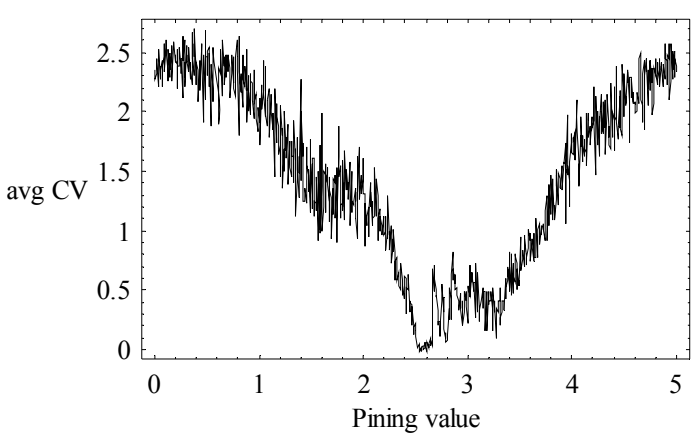

Figure 4: Development of $<\mathrm{M}>$-based Cost Function

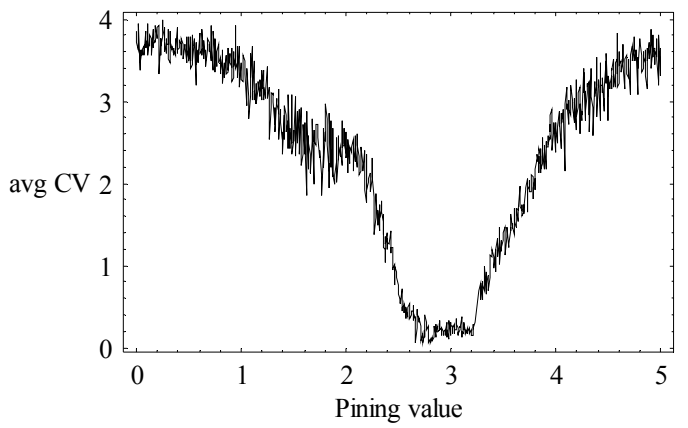

Figure 5: Development of $<\mathrm{M}>$-based Cost Function with no Target Pattern

\subsection{Cost Functions Comparison}

Results of 50 migration loops optimization are shown first, as all the cost functions should yield comparable results. The worst pinning value was found by the $<\mathrm{G}>$ based function, the best individual was identified by the mean mutual information based cost function, as can be seen from (Table 2). Approximate pinning values (PV) responding to the best and the worst individual can be seen in the last line.

Table 2: Comparison of Results for 50 Migrations

\begin{tabular}{|l|l|l|l|}
\hline $\begin{array}{l}\text { Cost } \\
\text { Function }\end{array}$ & $\begin{array}{l}\text { Best Avg } \\
\text { Individual }\end{array}$ & $\begin{array}{l}\text { Mean Avg } \\
\text { Individual }\end{array}$ & $\begin{array}{l}\text { Worst Avg } \\
\text { Individual }\end{array}$ \\
\hline $\begin{array}{l}\text { 1-norm } \\
\text { based }\end{array}$ & $1.476610^{-16}$ & $1.885610^{-16}$ & $2.387010^{-16}$ \\
\hline$<\mathrm{G}>$-based & $2.187110^{-16}$ & $3.187510^{-15}$ & $4.145810^{-14}$ \\
\hline$<\mathrm{M}>$-based & $2.109410^{-17}$ & $3.432410^{-16}$ & $1.199010^{-15}$ \\
\hline $\begin{array}{l}<\mathrm{M}>\text {-based } \\
\text { no target }\end{array}$ & $1.454410^{-16}$ & $1.838510^{-16}$ & $2.320410^{-16}$ \\
\hline \begin{tabular}{c} 
Best PV \\
\hline
\end{tabular} & 3.04017 & Worst PV & 3.2557 \\
\hline
\end{tabular}

As an illustration of these results follow figures of CML control by the worst and the best individual found (Figure 6).

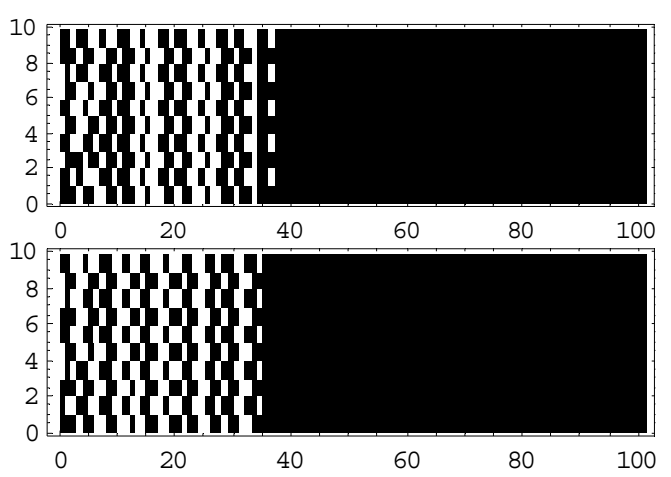

Figure 6: CML control by the best (top) and the worst (bottom) individual from 50 migrations

Along with the decreasing number of migration loops the efficiency variations became more apparent: the quality of $<\mathrm{G}>$-based cost function drastically decreased, $<\mathrm{M}>$-based cost functions quality also slightly decreased, while the fitness of individuals belonging to the 1 -norm based and untargeted $<\mathrm{M}>$ cost functions kept its level (Table 3).

Table 3: Comparison of Results for 20 Migrations

\begin{tabular}{|l|c|c|c|}
\hline $\begin{array}{l}\text { Cost } \\
\text { Function }\end{array}$ & $\begin{array}{c}\text { Best Avg } \\
\text { Individual }\end{array}$ & $\begin{array}{c}\text { Mean Avg } \\
\text { Individual }\end{array}$ & $\begin{array}{c}\text { Worst Avg } \\
\text { Individual }\end{array}$ \\
\hline $\begin{array}{l}\text { 1-norm } \\
\text { based }\end{array}$ & $1.476610^{-16}$ & $2.260410^{-16}$ & $5.606610^{-16}$ \\
\hline$<\mathrm{G}>$-based & $1.942810^{-16}$ & $1.487810^{-1}$ & 3.7195 \\
\hline$<\mathrm{M}>$-based & $1.454410^{-16}$ & $5.980810^{-15}$ & $9.791710^{-14}$ \\
\hline $\begin{array}{l}<\mathrm{M}>\text {-based } \\
\text { no target }\end{array}$ & $1.576510^{-16}$ & $1.895810^{-16}$ & $2.331510^{-16}$ \\
\hline \multicolumn{1}{c}{ Best PV } & 3.10329 & Worst PV & 4.73096 \\
\hline
\end{tabular}

The difference between the best and the worst individual, found by targeted $<\mathrm{M}>$ function and $<\mathrm{G}>$ function, respectively, can be easily seen in (Figure 7).

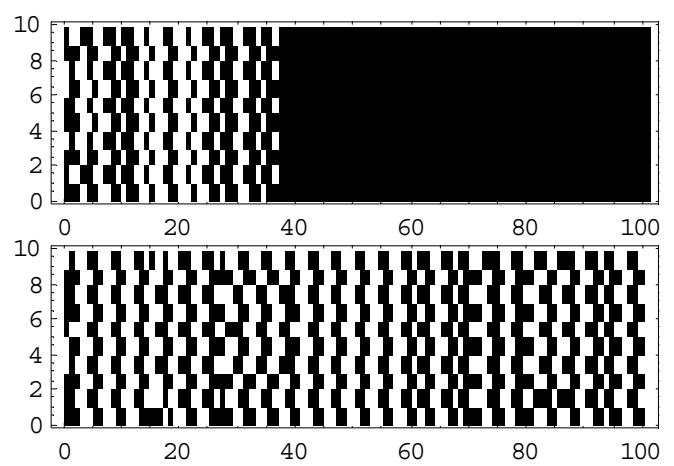

Figure 7: CML control by the best (top) and the worst (bottom) individual from 20 migrations

Individuals from the 10-migration batch became even more diverse as the quality of $\langle\mathrm{G}>$ and $<\mathrm{M}>$-based functions further decreased. On the other hand, untargeted $<\mathrm{M}>$ yielded individuals comparable to those of 1-norm (Table 4). 
Table 4: Comparison of Results for 10 Migrations

\begin{tabular}{|l|c|c|c|}
\hline $\begin{array}{l}\text { Cost } \\
\text { Function }\end{array}$ & $\begin{array}{l}\text { Best Avg } \\
\text { Individual }\end{array}$ & $\begin{array}{l}\text { Mean Avg } \\
\text { Individual }\end{array}$ & $\begin{array}{c}\text { Worst Avg } \\
\text { Individual }\end{array}$ \\
\hline $\begin{array}{l}\text { 1-norm } \\
\text { based }\end{array}$ & $1.310110^{-16}$ & $1.912710^{-16}$ & $4.041210^{-16}$ \\
\hline$<\mathrm{G}>$-based & $4.662910^{-17}$ & $4.585210^{-1}$ & 4.1921 \\
\hline$<\mathrm{M}>$-based & $4.329910^{-17}$ & $2.297710^{-11}$ & $5.437210^{-10}$ \\
\hline $\begin{array}{l}<\mathrm{M}>\text {-based } \\
\text { no target }\end{array}$ & $1.421110^{-16}$ & $2.545510^{-16}$ & $1.002510^{-15}$ \\
\hline \begin{tabular}{c} 
Best PV \\
\hline
\end{tabular} & 2.9725 & Worst PV & 0.806725 \\
\hline
\end{tabular}

There are two points that make the otherwise unsatisfying behaviour of $<\mathrm{G}>$-based cost function interesting: primo, it found the second best individual, and secundo, its worst pinning value drives CML into intriguing patterns, as can be seen from (Figure 8).

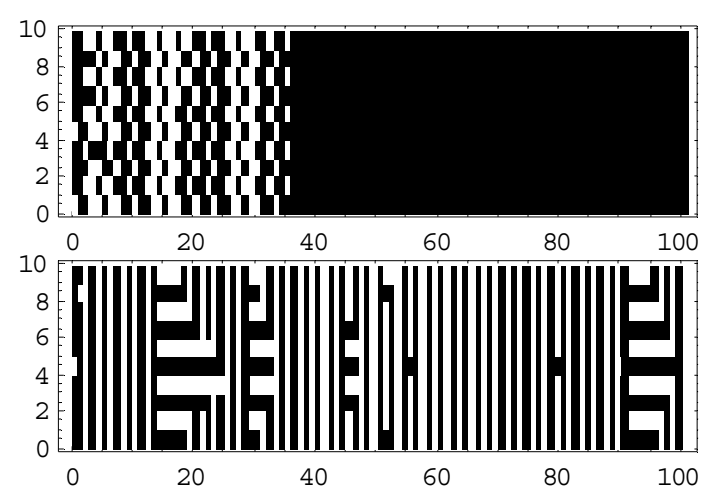

Figure 8: CML control by the best (top) and the worst (bottom) individual from 10 migrations

\section{CONCLUSIONS}

The main aim of this paper was to find out whether some of complexity measures, namely mean information gain $<\mathrm{G}>$ and mean mutual information $<\mathrm{M}>$, as described in (Wackerbauer et al., 1994), can be efficiently used in the control of deterministic chaos represented by the CML.

Four cost functions were constructed - cost function using 1-norm distance of tenth iteration of CML from the target state, cost function calculating $<\mathrm{G}>$ and $<\mathrm{M}>$ of tenth CML iteration joined to the vector TS, and the fitness based on $\langle\mathrm{M}>$ of tenth and eleventh iteration of CML. These functions were used in SOMA optimization, done for 10, 20 and 50 SOMA migration loops. Average fitness of the best, mean and worst pinning values found by each of cost functions was calculated, again using 1-norm distance.

Both the targeted $\left(\mathrm{CML}_{10}, \mathrm{TS}\right)$ and the untargeted $\left(\mathrm{CML}_{10}, \mathrm{CML}_{11}\right)$ mean mutual information cost functions proved to be able to keep up with the 1-norm cost function. $<\mathrm{G}>$-based function, on the other hand, failed to produce population of at least approximately identical fitness; some individuals even lead to a behaviour far from the T1S1 period. From all the alternatives to 1-norm cost function, untargeted $<\mathrm{M}>$ was found to be the best, being moreover unburdened by the necessity of knowledge of the fixed point of given equations and therefore more universal.

These results definitely prove that this topic asks for more thorough research as they indicate that the properties of mutual information could be utilised even in different optimization problems.

\section{ACKNOWLEDGEMENT}

This work was supported by the grant NO. MSM 7088352101 of the Ministry of Education of the Czech Republic and by grants of Grant Agency of the Czech Republic GACR 102/06/1132 and GACR 102/05/0271.

\section{REFERENCES}

Andrienko Yu.A., Brilliantov N.V., Kurths J. 2000. "Complexity of two-dimensional patterns", European Physical Journal B, vol. 15, pp 539 - 546

Greboki C., Lai Y.C. 1999. Controlling Chaos, In: Schuster H.G., Handbook of Chaos Control, WileyVch, ISBN 3-527-29436-8

Guanrong Chen. 2000. Controlling Chaos and Bifurcations in Engineering Systems, CRC Press, ISBN 0-8493-0579-9

Hilborn R.C. 1994. Chaos and Nonlinear Dynamics, Oxford University Press, ISBN 0-19-508816-8

Hu G., Xie F., Xiao J., Yang J., Qu Z. 1999. Control of Patterns and Spatiotemporal Chaos and its Application, In: Schuster H.G., Handbook of Chaos Control, Wiley-Vch, ISBN 3-527-29436-8

Li, Wentian. 1990. "Mutual Information Function versus Correlation Functions", Journal of Statistical Physics, vol. 60, pp $823-837$

Ott E., Greboki C., Yorke J.A. 1990. "Controlling Chaos", Phys. Rev. Lett. 64, 1196

Schuster H.G. 1999. Handbook of Chaos Control, Wiley-Vch, ISBN 3-527-29436-8

Shannon, C. E., 1948. "A Mathematical Theory of Communications", The Bell System Technical Journal, Vol. 27, pp $379-423,623-656$

Wackerbauer R., Witt A., Atmanspacher H., Kurths J., Scheingraber H. 1994. "A Comparative Classification of Complexity Measures" Chaos, Solitons \& Fractals, vol 4., no. 1., pp 133 - 173

Zelinka Ivan. 2004. SOMA - Self Organizing Migrating Algorithm",Chapter 7, 33 p. in: B.V. Babu, G. Onwubolu (eds), New Optimization Techniques in Engineering, Springer-Verlag, ISBN 3-540-20167X 
Zelinka Ivan. 2005. "Investigation on Evolutionary Deterministic Chaos Control - Extended Study" In: ECMS 2005, Riga, Latvia, in June 1-4, 2005

\section{AUTHOR BIOGRAPHIES}

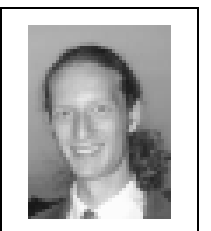

EDUARD NAVRATIL was born in the Czech Republic, reached his master degree in Mathematical Engineering at the Brno University of Technology in 2003. He is currently a Ph.D. student (information flow in complex systems and their control) at Tomas Bata University in Zlin. His e-mail address is: enavratil@fai.utb.cz

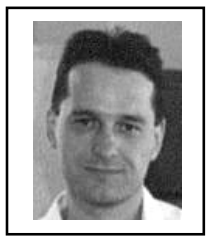

IVAN ZELINKA was born in the Czech Republic, and went to the Brno University of Technology, where he studied technical cybernetics and obtained his master degree in 1995. He obtained Ph.D. degree in technical cybernetics in 2001 at Tomas Bata University in Zlin. Now he is an associated profesor (artificial intelligence, theory of information) and head of department. His e-mail address is: zelinka@ft.utb.cz and his Web-page can be found at http://www.ft.utb.cz/people/zelinka.

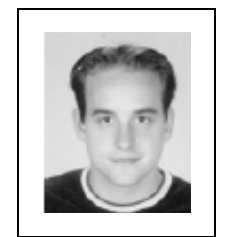

ROMAN SENKERIK was born in the Czech Republic, and went to the Tomas Bata University in Zlin, where he studied technical cybernetics and obtained his degree in 2004. $\mathrm{He}$ is now a Ph.D. student (control of chaos by evolutionary algorithms) at the same university. His e-mail address is:

senkerik@fai.utb.cz 